\title{
Notes sur la réception critique de le Corbusier en Grande-Bretagne, 1946-1972
}

Notes on the British Critical Reception of Le Corbusier 1946-1972

\section{Kenneth Frampton}

Traducteur : Sophie Jacotot

\section{(Q) OpenEdition Journals}

Édition électronique

URL : http://journals.openedition.org/crau/292

DOI : $10.4000 /$ crau. 292

ISSN : $2547-5746$

Éditeur

Éditions du patrimoine

Édition imprimée

Date de publication : 1 décembre 2009

Pagination : 20-42

ISBN : 978-2-85822-944-4

ISSN : $1296-4077$

\section{Référence électronique}

Kenneth Frampton, «Notes sur la réception critique de le Corbusier en Grande-Bretagne, 1946-1972 », Les Cahiers de la recherche architecturale et urbaine [En ligne], 24/25 | 2009, mis en ligne le 01 septembre 2017, consulté le 02 mai 2019. URL : http://journals.openedition.org/crau/292 ; DOI : 10.4000/crau.292 
Pour Kenneth Frampton, Rowe, Banham et Colquhoun, médiateurs de la pensée et des œuvres de Le Corbusier en Grande-Bretagne, jouèrent un rôle central dans le discours critique londonien de l'après-guerre, à partir du début des années 1970 . Mené de concert par des historiens, des critiques, des architectes, ce débat a permis de jeter des ponts entre théorie et pratique. Il révèle aussi qu'il existe une forme de critique incarnée par la réception d'une œuvre dans un projet nouveau. Frampton explore les conditions de réception de l'Unité d'habitation de Marseille, ainsi que les échanges autour du classicisme latent de Le Corbusier (révélé par les analyses de Rowe) et de la réinterprétation du vernaculaire dans ses projets des années 1950. II souligne l'écho de ces questions sur la lisibilité de l'architecture par le citoyen, débat important dans le contexte de la construction des villes nouvelles et dans le climat social-démocrate britannique.

\section{Notes \\ sur la réception critique de Le Corbusier en Grande-Bretagne} 1946-1972

KENNETH FRAMPTON

"Lorsque la critique - quand elle parle de littérature - émet un discours parallèle sur la forme et le destin d'une culture dans son ensemble, sa voix attire largement l'attention. Lorsque la "culture" est devenue une question politique de premier plan, la "poésie" une métaphore de la qualité de la vie en société, et le langage un paradigme des pratiques sociales dans leur ensemble, la critique a pu alors revendiquer sérieusement le droit d'exister. Aujourd'hui, à l'exception de son rôle marginal dans la reproduction des relations sociales dominantes dans les milieux académiques, elle est presque entièrement privée d'une telle raison d'être. Elle ne s'engage de manière significative dans aucune question sociale substantielle et constitue un type de discours presque entièrement tourné vers l'autoévaluation et l'autoreproduction. Doit-on en conséquence laisser la critique mourir ainsi, ou peut-on lui découvrir un rôle plus productif?»

Terry Eagleton, The Function of Criticism, $1984^{1}$

Quatre personnalités assez inattendues ont été les premiers médiateurs de la réception de Le Corbusier en Grande-Bretagne dans la

1. Terry Eagleton, The Function of Criticism: From «The Spectator » to Post-structuralism, Londres, Verso, 1984. 
période de l'après-guerre. II s'agit, d'une part, de deux membres de l'intelligentsia allemande en exil à Londres dans la seconde moitié des années 1930, Nikolaus Pevsner et Rudolf Wittkower et, d'autre part, de deux de leurs premiers élèves en histoire de l'architecture, respectivement Reyner Banham et Colin Rowe. Alors que Banham avait été formé comme ingénieur aéronautique avant d'entrer à l'Institut Courtauld pour suivre I'enseignement de Pevsner, Rowe avait reçu une formation d'architecte à l'université de Liverpool avant d'étudier à l'Institut Warburg auprès de Wittkower, sous la direction duquel il écrivit une thèse sur Inigo Jones. Tous les quatre jouèrent un rôle central dans le discours critique développé à Londres de 1946 à 1972, Banham étant la cheville ouvrière de ce débat culturel, notamment à partir de son entrée au comité de rédaction de The Architectural Review en 1953. C'est lui qui défendit la cause des New Brutalists ${ }^{2}$, à la suite de l'émergence de ce courant dans le sillage de l'exposition This is Tomorrow, présentée à la Whitechapel Art Gallery de Londres en 1956 et proposée par le mouvement Independent Group de I'ICA (Institute of Contemporary Arts). Bien que James Stirling ait été membre du groupe et qu'il ait participé à l'exposition, il n'en conserva pas moins certaines affinités avec la position plus aristocratique de Colin Rowe qui, à cette date, enseignait déjà aux États-Unis. Même si elle était informelle, I'association Stirling/Rowe constituait l'un des épicentres de la pensée architecturale à Londres, tandis que l'autre pôle était centré sur Banham qui, avec Alison et Peter Smithson, tendait vers une architecture théoriquement plus populaire et technologique.
Au-delà des nuances subtiles qui distinguaient les factions à cette époque, ce qui est particulièrement marquant c'est la manière dont les critiques et les architectes praticiens ont contribué de concert à ce débat, faisant fi des générations: dans la génération d'avantguerre il y avait des personnalités comme John Summerson, Berthold Lubetkin, Robert Furneaux-Jordan, James Maude Richards et Lionel Brett, mais également d'autres comme Trevor Dannat, Thomas Stevens, Oliver Cox, Ian Nairn et Alan Colquhoun qui ont émergé sur la scène architecturale depuis la guerre. En jetant des ponts entre théorie et pratique, ce débat était assez comparable, quoique dans une veine moins idéologique, à ce qui se passait au Centro Studi d'Ernesto Nathan Rogers, très actif à Milan à la même époque, gravitant autour de la revue Casabella dont Rogers était le directeur, et élaborant un discours critique développé dans les années 1960 par ses principaux acolytes, Vittorio Gregotti, Aldo Rossi, Guido Canella et Giorgio Grassi ${ }^{3}$. En Italie, le débat était animé par des architectes praticiens - à la différence de ce qui se passait en Angleterre, le discours critique y était perçu comme nécessairement articulé à la pratique, ce qui, rétrospectivement, fait de Londres une scène d'autant plus remarquable. En se reportant plus d'un demi-siècle en arrière, on peut voir cette période comme un entre-deux social-démocrate fécond entre la fin de la Seconde Guerre mondiale et la suspension du système de Bretton Woods en 1971, tournant à partir duquel l'État-providence a commencé à être ébranlé par les forces du capitalisme mondialisé. Notons que cet événement d'ordre politique coïncide avec le plan pour Milton Keynes (la dernière des villes nouvelles
2. Reyner Banham, The New Brutalism: Ethic or Aesthetic?, Londres, The Architectural Press, 1966; version française: Le Brutalisme en architecture. Éthique ou esthétique, Paris, Dunod, 1970.
3. Voir dans ce dossier la contribution de Pierre-Alain Croset et Michele Bonino intitulée "Casabella 1982-1996. Autour de Vittorio Gregotti et du "réalisme critique" en architecture ". 
britanniques), conçu en 1969 par l'équipe LlewelynDavies, Weeks, Forestier-Walker et Bor, et élaboré comme une grille "sans lieu », délibérément superposée aux paysages bucoliques au tracé organique du comté de Buckinghamshire ${ }^{4}$.

\section{Clive Entwistle et le concours pour le Crystal Palace (1946)}

L'organisation du concours international pour la rénovation du site du Crystal Palace, faisant suite à la destruction de l'édifice par le feu en 1937, fut la cause célèbre de la scène architecturale londonienne dans l'immédiat après-guerre. Le projet le plus ouvertement néocorbuséen pour ce concours était celui que proposait Clive Entwistle en collaboration avec l'ingénieur Ove Arup. II rassemblait, fait remarquable à cette date, des éléments tirés du travail de Le Corbusier entre la fin des années 1920 et le début des années 1930, notamment son Musée mondial pyramidal de 1929, les salles groupées de son projet de Palais des Soviets de 1931, et sa proposition de stade à ciel ouvert pour 100000 spectateurs de 1937. Inspirée du plan axial de la Cité mondiale - Mundaneum - de Le Corbusier en 1929, la mégastructure d'Entwistle était encore plus monumentale, avec sa longue plate-forme à arcades qui unifiait la composition. Notons que Le Corbusier commentera favorablement ce projet dans le second volume d'Architects Year's Book de Trevor Dannat en 1947, année durant laquelle Le Corbusier donna un cours à l'Architectural Association de Londres, à l'invitation des étudiants, parmi lesquels Oliver Cox, Clive Entwistle, Joseph Rykwert et Paffor Keating Clay.
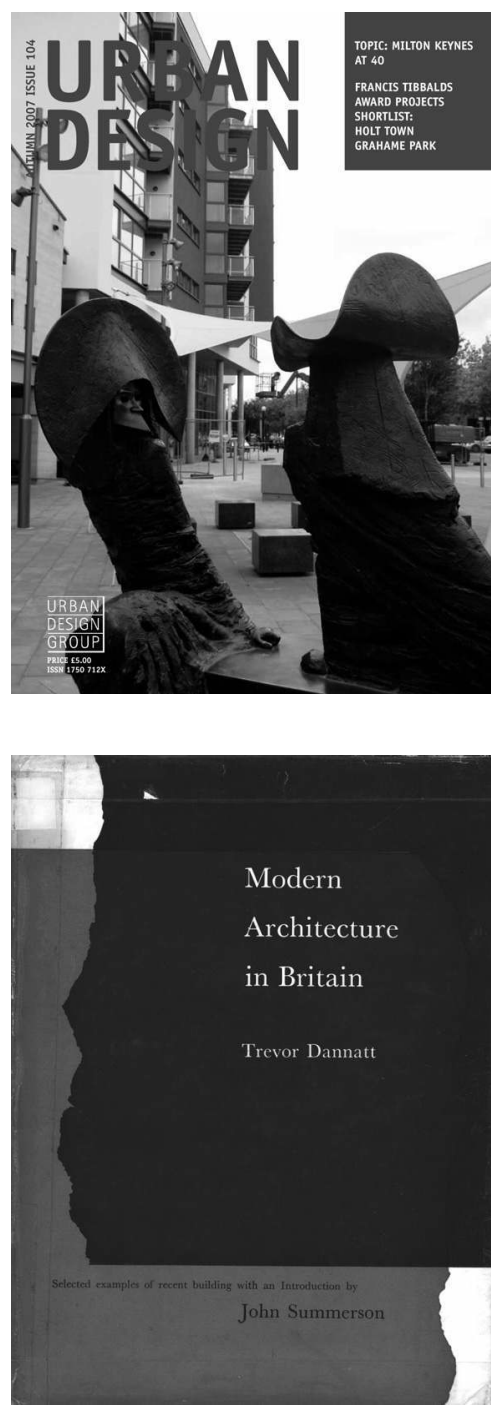

Urban Design, $n^{\circ} 104$, automne 2007. Dossier «Milton Keynes at $40 »$, pp. 10-41.

Couverture

du livre de Trevor Dannatt, Modern Architecture in Britain, Londres, 1959. 


\section{L'héritage de Lubetkin, 1945-1956}

Le style néocorbuséen de Berthold Lubetkin dans l'immédiat avant-guerre eut une influence directe sur la production architecturale britannique durant la seconde moitié des années 1940, ce dont témoigne le projet de Leslie Martin et Peter Moro pour le Royal Festival Hall, réalisé en 1951 comme une démonstration tour de force du plan libre, en particulier tel qu'il figurait, à une autre échelle, dans la proposition de Le Corbusier pour le concours du Palais des Soviets en 1931. Le foyer à plan libre du Royal Festival Hall revisitait dans le détail la syntaxe du Finsbury Health Center de l'agence Tecton (fondée par Lubetkin) en 1938, sur lequel Moro avait travaillé au début de sa carrière d'architecte. Cependant, ce ne fut pas le seul édifice de la période à subir l'influence de Lubetkin ; on la retrouve ainsi dans le Brynmawr Rubber Factory construit en 1952 dans le sud du Pays de Galles, à partir du projet d'Architects Co-Partnership associé à Arup, et dans l'édifice du Trades Union Congress Memorial Building à Londres, réalisé par David du Rieu Aberdeen en 1957. On pourrait encore signaler les plans néo-corbuséens de Lubetkin pour la ville nouvelle, non réalisée, de Peterlee dans le comté de Durham, en 1950.

La complexité des relations entre Le Corbusier et Lubetkin peut s'expliquer en partie par leur sensibilité commune aux théories socialistes utopiques du $X I X{ }^{e}$ siècle. Ces influences se sont manifestées de différentes manières, dans les théories urbanistiques de Le Corbusier et dans les projets architecturaux révolutionnaires pour la Russie du milieu des années 1920; de même que leurs positions rivales se révèlent dans Vers

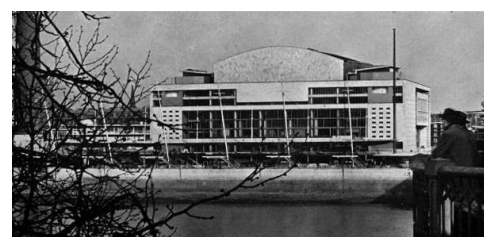

\begin{abstract}
J. Leslie Martin, Royal Festival Hall, South Bank, Londres, 1951. Façade vue depuis la Tamise. (C) Galwey, Architectural Review.
\end{abstract}

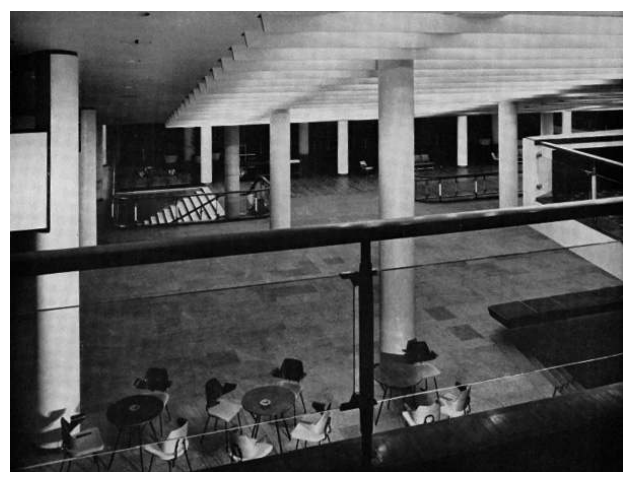

Royal Festival Hall, vue du foyer principal. (C) Mann 
une architecture de Le Corbusier en 1923 et dans Style and Epoch de Moisei Ginzburg en 1924. Pur produit de la révolution d'Octobre, vivant dans le Paris cosmopolite des années 1920, Lubetkin était particulièrement attiré par le Plan Voisin de Le Corbusier, exposé pour la première fois dans son pavillon de l'Esprit Nouveau en 1925. On sait que, durant ses cinq premières années de pratique à Londres, Lubetkin a admiré Le Corbusier sans équivoque; paradoxalement, leurs relations commencèrent à se dégrader à la suite de l'approbation enthousiaste par Le Corbusier de la première œuvre de Lubetkin en Angleterre, les appartements Highpoint One, réalisés en 1935. Des divergences politiques radicales sont à la base de ce schisme - socialisme d'une part et fascisme de l'autre - mais Lubetkin sera toute sa vie influencé par les logements à redents du Plan Voisin et par le concept d'habitat collectif tel qu'il a été mis en œuvre par Le Corbusier dans le Pavillon Suisse de 1933 et dans l'idée de la maison commune, ou dom kommuna, réalisée par Ginzburg dans l'immeuble Narkomfin à Moscou en 1932. Cependant, en dernière analyse, c'est le vaste espace vert de la Cité radieuse de Le Corbusier, déjà manifeste dans sa Réponse à Moscou de 1930, qui restera comme I'une des aspirations les plus durables de Lubetkin dans

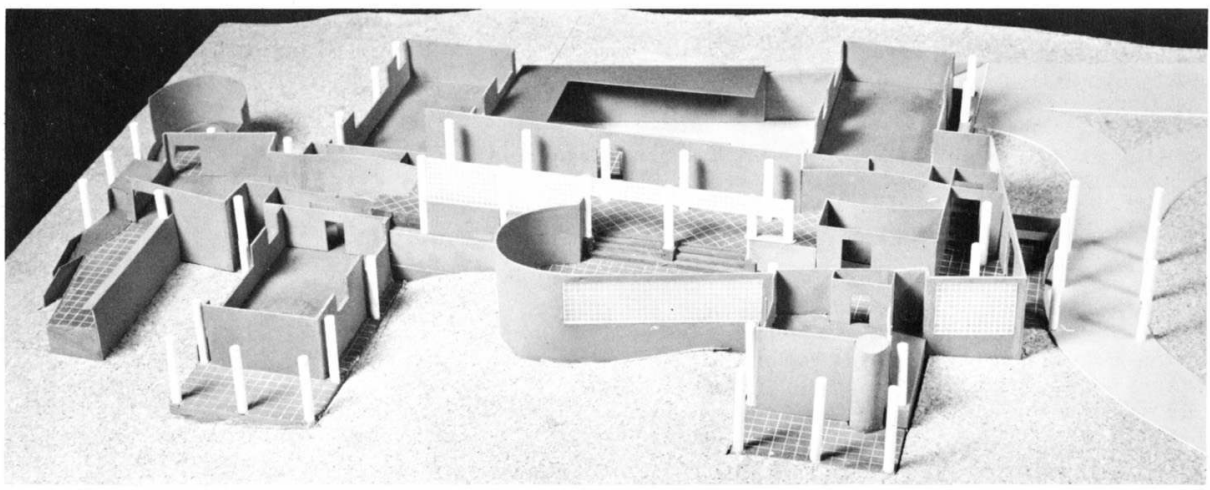

Immeuble Highpoint

One, 1935-1938:

maquette et plan du rez-

de-chaussée. Architectes

Berthold Lubetkin

et Tecton.

(C) Archive Berthold Lubetkin (RIBA).

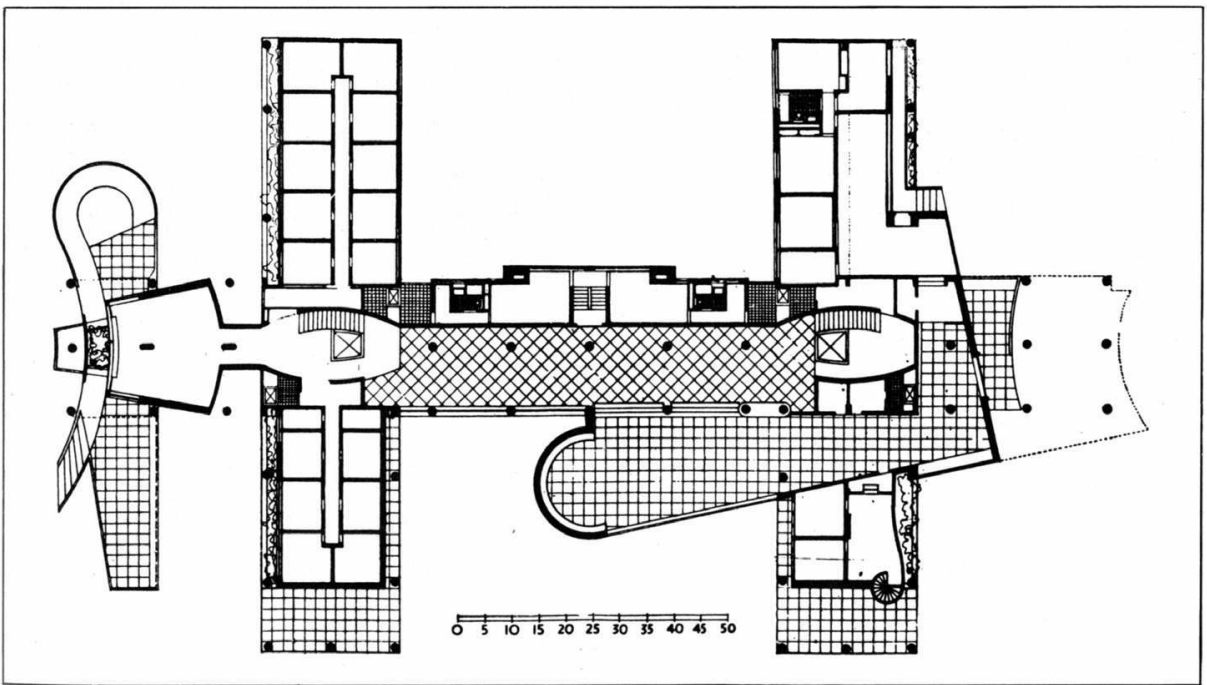


tous ses projets de logements ouvriers réalisés aprèsguerre dans le quartier de l'East End de Londres, en commençant par le Spa Green Estate (groupe Tecton) à Finsbury en 1950. Une « trace » de la Cité radieuse de Le Corbusier est sans doute encore décelable dans le traitement de l'élévation de l'immeuble Spa Green, avec la scission entre son « dos » en brique et sa façade carrelée rappelant la forme du Pavillon Suisse. On retrouve un tel maniérisme, déjà perceptible dans l'immeuble Highpoint Two de Lubetkin en 1936, dans l'élévation de tous les logements réalisés par l'équipe Tecton en GrandeBretagne entre 1950 et 1967. Cette tendance à transcender le fonctionnalisme par la modénature incluait une part de formalisme que Lubetkin dénoncera plus tard comme une faiblesse inhérente aux constructivistes russes des années 1920; il le fera dans deux essais successifs sur l'architecture soviétique publiés dans The Architectural Association Journal en 1956 ${ }^{5}$. Alors qu'il était en quête d'une syntaxe capable de conférer une urbanité hiérarchisée à la répétition de modules, telle qu'on peut la trouver dans le Paris d'Haussmann, Lubetkin ignora délibérément la réponse de Le Corbusier à ce défi, incarnée dans l'Unité d'Habitation réalisée à Marseille en 1952.

Le seul élément partagé par Lubetkin et Le Corbusier était leur intérêt pour la création d'un espace public à
Spa Green Estate,

Finsbury, Londres, 1950:

maquette. Architectes

Berthold Lubetkin et Tecton.

(c) Archive Berthold

Lubetkin (RIBA).
5. Berthold Lubetkin, "Soviet Architecture: Notes on Developments from 1932 to 1955 », Architectural Association Journal, 72, septembre 1956.
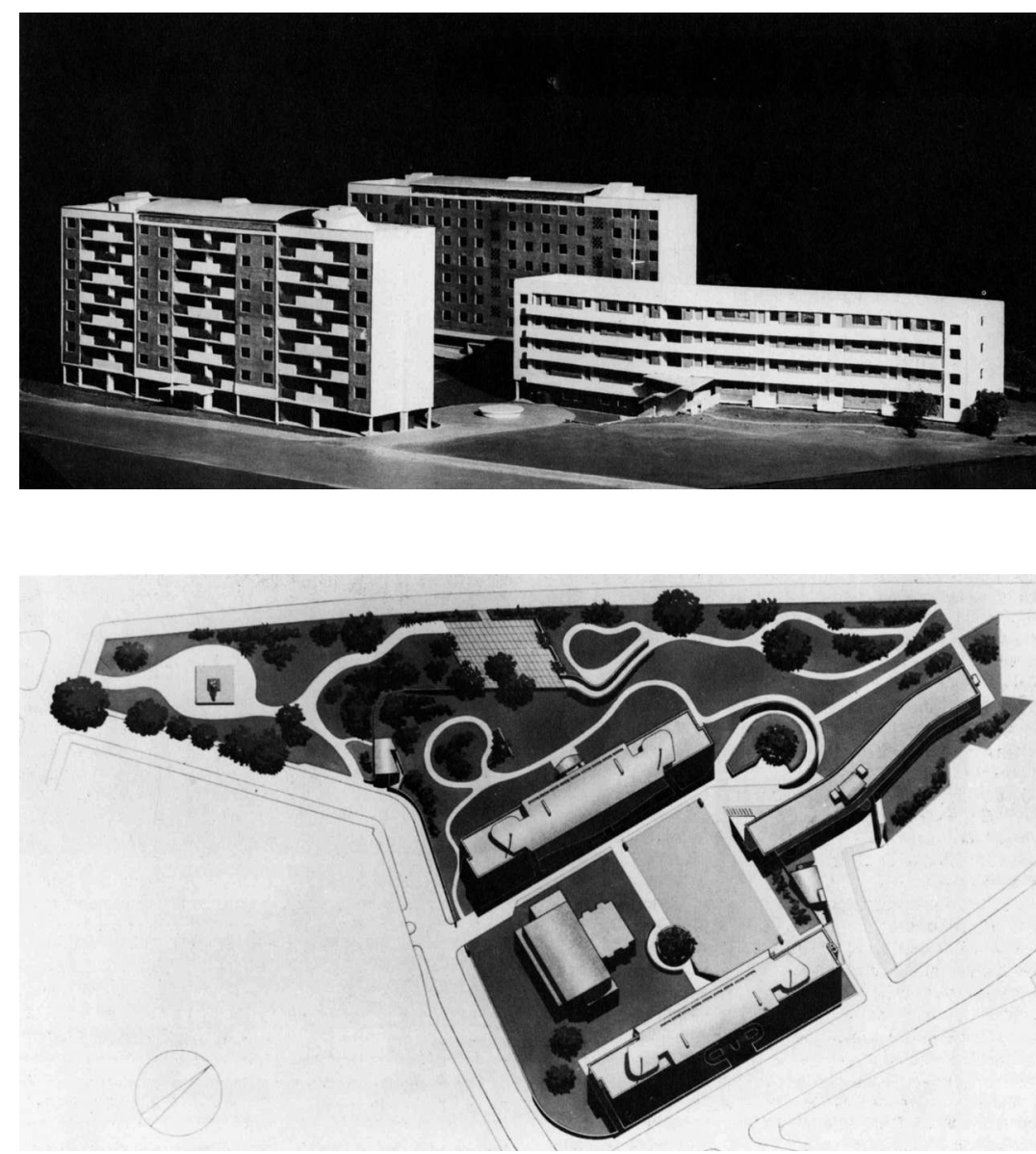
grande échelle, adapté à la collectivité « héroïque » de l'époque moderne. C'est ce qui mènera Lubetkin, à la suite de sa visite en Russie en 1953, à se prononcer en faveur du réalisme socialiste, notamment pour la façon dont ce courant se démarquait radicalement de l'ethos petit-bourgeois du parc urbain des premières New Towns, ces villes nouvelles bâties sous les auspices du gouvernement travailliste d'après-guerre, dont celle d'Harlow dans le comté de l'Essex, par Frederick Gibberd, est un bon exemple. Avec cela présent à l'esprit, le deuxième essai de Lubetkin sur l'architecture soviétique se termine par ces mots: "Enserrés dans les pages sans rapport d'un catalogue monumental de maçon, quelques édifices soviétiques (mais pas tous) sont pourtant capables - grâce à des plans solidement conçus, à un usage prodigieux d'espaces ouverts, à des échelles à couper le souffle - de produire de vastes ensembles ordonnés dont l'impact est difficilement oubliable pour un architecte occidental, dans une période de fragmentation pittoresque et de "développement mixte". [...] Avec tant de choses derrière eux et plus encore devant eux, les architectes soviétiques peuvent sans doute dire avec Eluard: "Je ne regrette rien, j'avance" ${ }^{\prime 6}$. "

Cette conception d'une grande structure résidentielle incluant des espaces verts à une échelle héroïque était ce que Le Corbusier et Lubetkin avaient en commun, comme

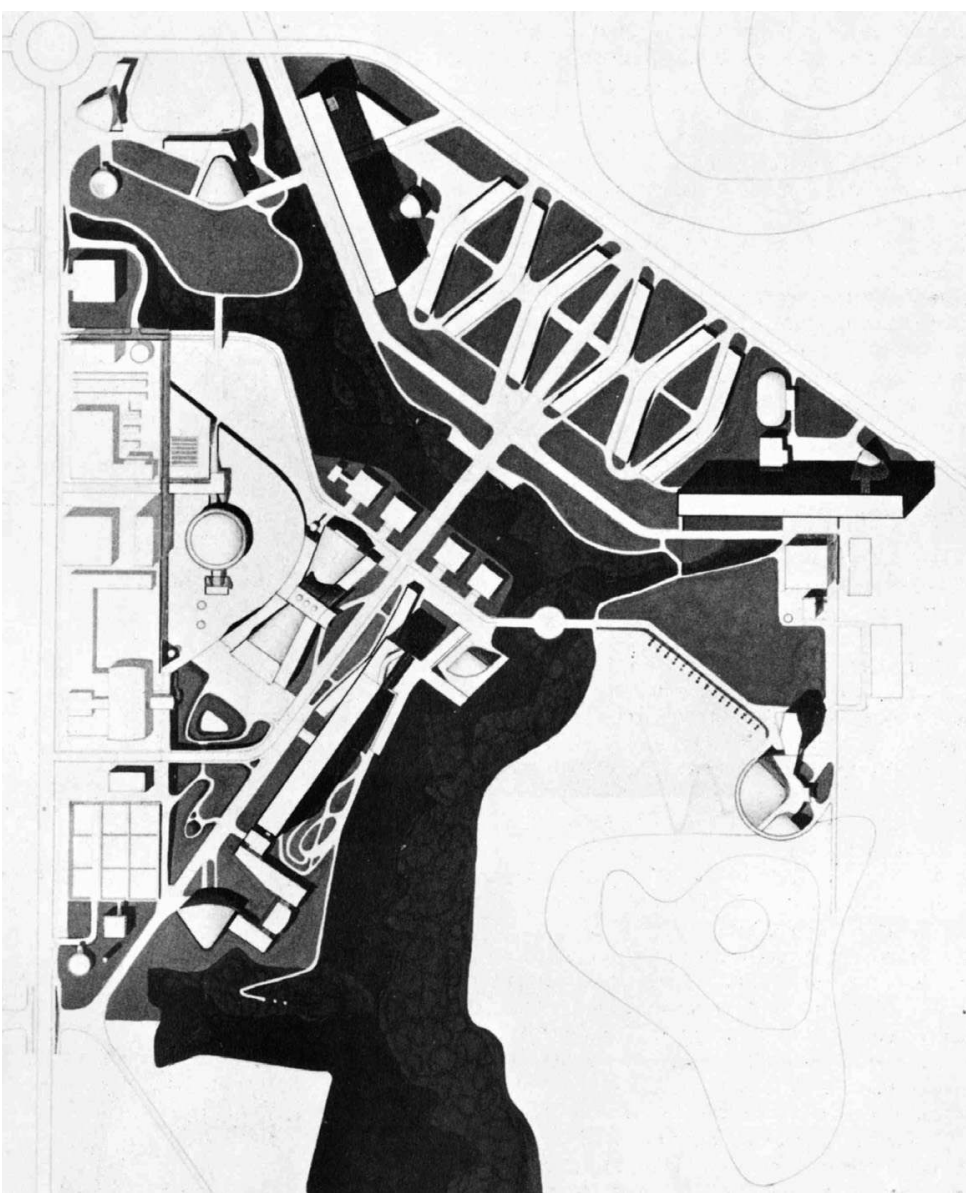

Plan masse pour la ville nouvelle de Peterlee, 1948-1950. Architecte Berthold Lubetkin. (C) Archive Berthold Lubetkin (RIBA). 
on peut le voir dans les méga-immeubles de douze étages agencés avec un vaste espace vert, qui constituent le cœur du projet de Lubetkin pour Peterlee en 1950.

\section{Wittkower, Rowe et le néo-palladianisme britannique, 1947-1965}

Ayant reçu une rigoureuse formation intellectuelle en Allemagne, à Munich et Berlin, puis enseigné à Londres à I'Institut Warburg - où Colin Rowe devint son élève en 1945 -, Rudolf Wittkower pouvait difficilement imaginer à quel point ses Architectural Principles in the Age of Humanism $^{7}$ de 1949 allaient influencer la scène théorique britannique à travers I'essai de Rowe en 1947, "The Mathematics of the Ideal Villa », qui appliquait la thèse de Wittkower à la villa Stein à Garches. Pour la première fois, cet essai révélait le potentiel formel du principe du plan libre de Le Corbusier. Rowe faisait la démonstration de façon non seulement révélatrice mais aussi surprenante (car personne ne les avait remarquées jusque-là) des correspondances rythmiques et harmoniques entre la villa Malcontenta d'Andrea Palladio (1560) et la villa Stein de Le Corbusier (1927). Sans avoir eu un impact immédiat sur la pratique britannique d'après-guerre, ceci permit de démontrer, par défaut, que le principe du plan libre avait été très peu utilisé, et encore moins compris, dans l'architecture britannique néo-corbuséenne de la période d'avant-guerre, ce que l'ouvrage de Francis Reginald Stevens Yorke, The Modern House in England, publié en 1937, tendait à accréditer. La sophistication de la relecture de Le Corbusier par Rowe, à travers un prisme académique, entérinait le classicisme latent de Vers une architecture, traduit pour la première fois en anglais en
1927, un trope humaniste qui tempérait sa réputation de radicalisme intransigeant. La thèse de Rowe révélait les correspondances suivantes: "La villa de Garches et la villa Malcontenta sont toutes deux conçues comme des pavillons uniques; et, mis à part des variantes dans le traitement du toit, elles ont toutes deux des proportions équivalentes, chaque édifice mesurant 8 unités de longueur pour 5 1/2 de profondeur et 5 de hauteur. Au-delà, on peut observer une structure en travée comparable. Chaque maison présente (et dissimule) un rythme alterné de modules simples et doubles, et possède, si on l'observe depuis la façade vers l'arrière, une distribution tripartite des points d'appui. Mais à ce sujet, mieux vaut introduire un "presque" car si la distribution des ensembles horizontaux est, dans les deux cas, à peu près la même, il y a des différences non négligeables dans la distribution de ces points d'appui sur un plan vertical. À Garches, depuis la façade jusqu'à l'arrière, l'intervalle spatial fondamental procède d'un rapport de $1 / 2: 1 \frac{1 / 2: 1}{1} 1 / 2: 1 \frac{1}{2}: 1 / 2$, tandis que dans la Malcontenta on observe la séquence $2: 2: 1$. En d'autres termes, grâce à l'usage d'unités en porte-à-faux, Le Corbusier obtient une réduction de la travée centrale au profit d'autres espaces, tandis que Palladio assure la prééminence de la division centrale grâce à une progression vers le portique qui concentre l'attention sur ces deux espaces. Le premier schéma est donc potentiellement étendu et sans doute égalitaire, et l'autre est concentrique et assurément hiérarchique, mais au-delà de ces différences, un élément saillant - terrasse en débord ou portique fixé - occupe dans les deux cas 1 unité $1 / 2$ de profondeur ${ }^{8} \ldots »$
7. Rudolf Wittkower, Architectural Principles in the Age of Humanism, Studies of the Warburg Institute, vol. XIX, Londres, 1949; version française: Les Principes de l'architecture à la Renaissance, trad. de Claire Fargeot, Paris, Les Éditions de la Passion, 1996.
8. Colin Rowe, "The Mathematics of the Ideal Villa », The Architectural Review, $n^{\circ} 101$, mars 1947, p. 3-4. Version française: "Mathématique de la villa idéale", Mathématiques de la villa idéale et autres essais, Paris, Hazan, 2000, p. 10-11. 
Quasiment aucun architecte parmi les contemporains de Rowe ne savait comment s'approprier cette idée, pas même James Stirling, récemment démobilisé, dont Rowe avait été le professeur à la faculté d'architecture de l'université de Liverpool, de 1947 à 1950. Rowe compléta la thèse de Wittkower grâce à sa connaissance du cubisme, acquise probablement aux côtés de Thomas Stevens avec qui il avait étudié à Liverpool, après avoir été réformé de l'armée britannique en 1942. Le fait que le cubisme ait servi de point de départ au purisme de Le Corbusier et d'Amédée Ozenfant en 1918 a sans doute encouragé Rowe à considérer les compositions frontales de la villa Stein comme la mise à plat de la forme palladienne.

La lecture de Le Corbusier par Rowe à travers le prisme du palladianisme de Wittkower survint à la fin des années 1940, dans un contexte où la Renaissance venait d'être réévaluée en des termes maniéristes par Geoffrey Grigson, Anthony Blunt et Nikolaus Pevsner. Rowe manifesta sa sensibilité à cette mutation dans son essai de 1950 sur le maniérisme et l'architecture moderne ${ }^{9}$, tout comme son penchant pour le baroque nourrit son étude sur le Churchill College, dont le concours fut organisé en 1959, avec l'intitulé "Blenheim of the Welfare State ». Dans cette étude, Rowe comparait le travail de James Gibbs à Cambridge au formalisme contrapuntique de la proposition de Stirling et Gowan pour ce concours. II convient de noter également un certain néo-palladianisme dans le projet par ailleurs néo-miesien des Smithson pour la Hunstanton School de 1950 (comté de Norfolk). Deux ans plus tard, toutefois, ils se rallieront au slogan « Mies est grand mais Corb est parlant ${ }^{10}$ ».

\section{L'Unité d'Habitation et la street-in-the-air, 1952-1959}

L'Unité d'Habitation de Marseille (1947-1952) eut un impact profond sur la scène architecturale britannique, ce qui apparaît clairement avec le concours pour l'ensemble de logements de Golden Lane en 1952, dans la mesure où deux traits fondamentaux de l'Unité - la rue intérieure et le principe des appartements en duplex imbriqués - ont été transposés dans deux des projets soumis au concours: celui d'Alison et Peter Smithson et celui de Jack Lynn et Ivor Smith, alors encore étudiants. Les deux propositions remplaçaient le large corridor intérieur de I'Unité par une rue-en-I'air (street-in-the-air), présentée comme une rue-pont (street deck). Dans les deux cas, les unités imbriquées de Le Corbusier faisaient place à une combinaison de résidences à deux étages et d'appartements. Notons que ces propositions jumelles imitaient et déniaient conjointement certains aspects découlant du concept de l'Unité : tout d'abord, l'idée initiale de Le Corbusier de relier ses immeubles " à redents", afin de constituer une structure résidentielle qui crée un espace et ensuite, son projet d'après-guerre d'espace urbain qui trouve son aboutissement dans la répétition sérielle des unités d'habitation au sein d'un espace vert continu, comme dans son plan pour la reconstruction de Saint-Dié en 1945. Tandis que le concept de redent réapparaît comme une continuité articulée dans les projets précités pour Golden Lane, où les immeubles sont reliés par des rues-en-l'air horizontales, il n'engendre pas alors le type d'espace collectif qui avait été envisagé de façon indépendante par Le Corbusier et par Lubetkin.

9. C. Rowe, "Mannerism and Modern Architecture ", The Architectural Review, no 107, mai 1950; trad. fr. dans Mathématiques de la villa idéale [...], op. cit. note 8, pp. 37-72. 
Au neuvième Congrès international d'Architecture moderne (CIAM 9) à Aix-en-Provence, en 1953, les Smithson ont reconnu leur dette envers le principe du redent, tout en prenant leurs distances avec I'Unité : «Dans le contexte de la grande ville avec des édifices élevés, nous proposons, afin de rester libre de ses mouvements, une ville à plusieurs niveaux avec des "rues-enI'air" résidentielles. Celles-ci sont reliées par un complexe continu sur plusieurs niveaux [...]. Ce concept est en opposition directe avec l'isolement arbitraire des soi-disant communautés de l'“Unité" et du voisinage $^{11}$ !...»

Le complexe résidentiel Park Hill de Lynn et Smith, réalisé à Sheffield en 1961, révèle les contradictions de la « rue-en-l'air »: tout d'abord, les accès surélevés ne pouvaient égaler, en termes phénoménologiques ou sociaux, les qualités éprouvées d'une rue au niveau du sol; ensuite, la galerie d'accès à ciel ouvert constituait, en raison du climat anglais, un mode d'approche particulièrement inapproprié la majeure partie de l'année. L'aversion de longue durée de Reyner Banham vis-à-vis de Le Corbusier ne s'est probablement jamais exprimée aussi clairement que lorsqu'il fit précéder, en 1962, son évaluation négative de l'Unité de Marseille par un jugement positif sur Park Hill.

Une décennie plus tôt, I'Unité avait été accueillie avec enthousiasme par l'un des groupes d'architectes du département du logement du « London County Council » (LCC), le Conseil métropolitain de Londres, dont le débat avait donné lieu à une critique publiée dans The Architectural Review, en mai 1951. William Howell y faisait l'éloge de l'Unité, considérée comme le prototype d'une

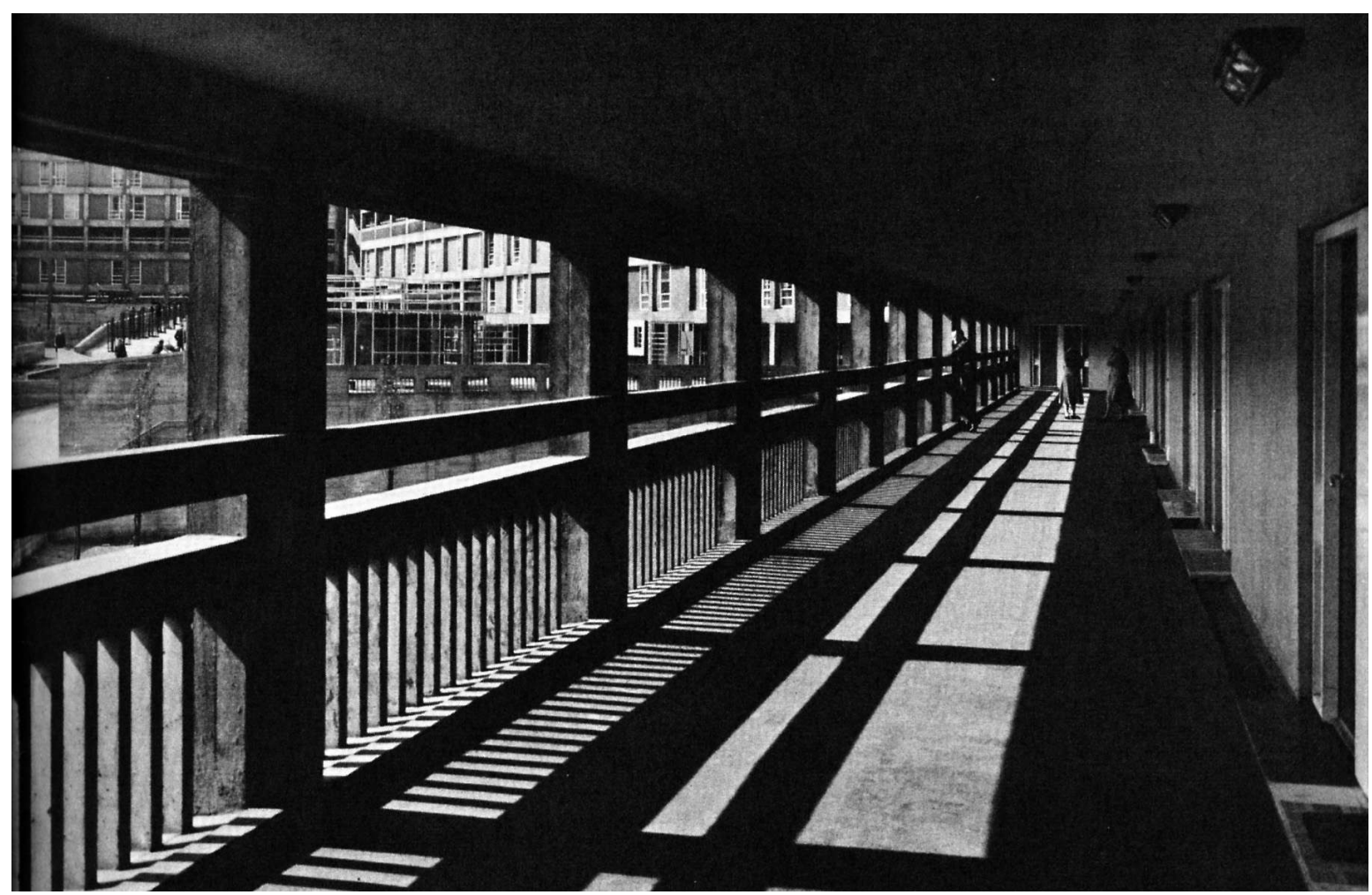




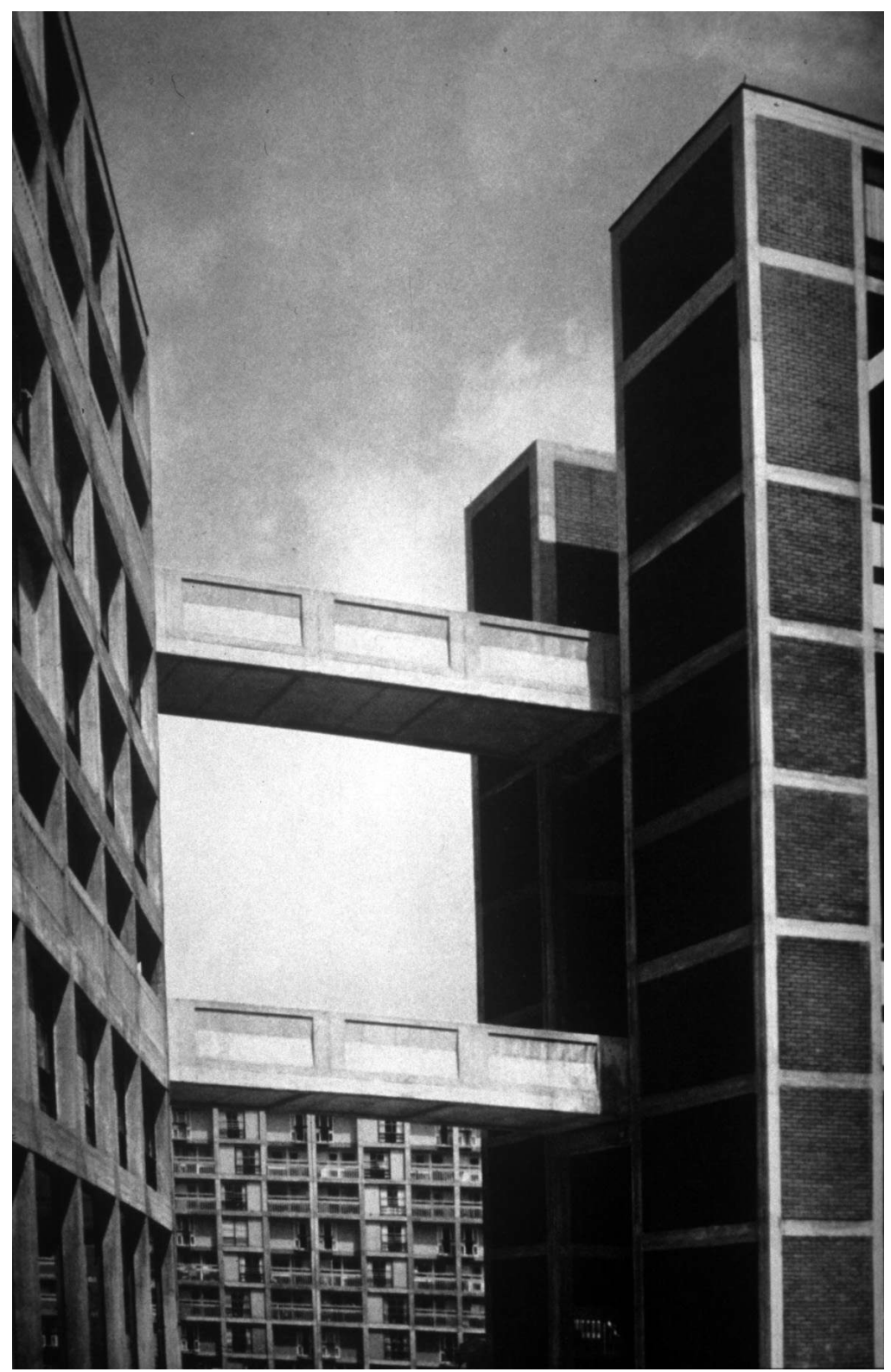

Park Hill, Sheffield, 1961 :

tour de l'ascenseur

et passerelles piétonnes.

Architectes J. Lewis

Womersley, Jack Lynn,

Ivor Smith, Frederick

Nicklin.

(C) Architectural Review,

De Burgh Galey.

Page de gauche: Park Hill, Sheffield, 1961 :

passerelles piétonnes.

(C) Architectural Review,

Reyner Banham. 
forme beaucoup plus complète de développement urbain, déjà présente dans le projet de Le Corbusier pour Saint-Dié en 1945. Comme Howell le souligne alors: « $\mathrm{Ce}$ projet d'après-guerre de Le Corbusier pour reconstruire une petite ville des Vosges représente le développement le plus récent de ses idées sur l'urbanisme. Dans le plan, Le Corbusier présente huit Unités, étroitement reliées à de larges voies piétonnes menant vers et à travers une série de "piazzas" [...]. Toute la circulation automobile est isolée sur différents niveaux. Les huit Unités forment une série de rues verticales à quelques minutes à pied du centre-ville, et sont situées dans un parc paysager avec des écoles installées tout autour. À partir de ce centre de forte densité (autour de 250 personnes par acre), de longs rubans de maisons basses s'étendent vers la campagne le long d'allées paysagères, qui sont séparées des principales routes menant à la ville. Les deux, la rue verticale reliée à la piazza et la rue horizontale rayonnant vers la campagne, sont clairement différenciées dans le plan, chacune correspondant à une interprétation ingénieuse $d^{\prime}$ une certaine manière de vivre ${ }^{12}$. "

L'analyse par William Howell du prototype de l'Unité comme élément clé de la proposition de Le Corbusier pour Saint-Dié laissait présager sa future collaboration avec Gillian Howell sur le projet pour le LCC - un bloc de onze étages bâti dans les faubourgs Lambeth et Rotherhithe de Londres - et, plus tard, dans la ville nouvelle de Roehampton, entre 1952 et 1959. La structure en duplex de l'Unité a été adoptée comme point de départ pour la dimension verticale de ce prototype, ce que confirme l'immeuble de onze étages de Bentham Road à Hackney, réalisé en 1955, à partir des projets d'Alan Colquhoun,

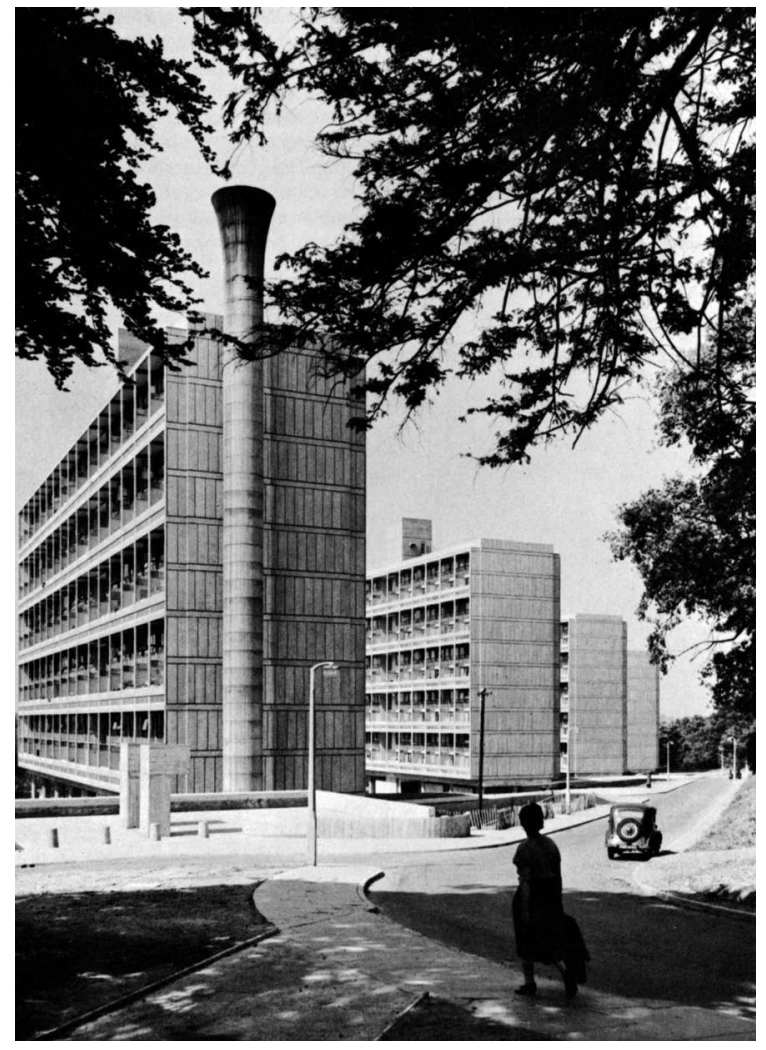

Maisonnette Slab Block, Alton Estate West, Roehampton. London County Council Housing Department, architectes Colin Lucas, John Partridge, Sanley Amis, William Howell, John Killick, 1955.

(c) London County Council.
11. Alison Smithson, The Emergence of Team X out of CIAM (Documents), Londres, Architectural Association, 1982, p. 7.

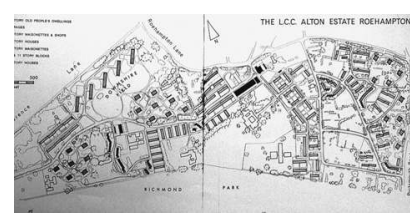

Alton Estate, Roehampton: plan du London County Council Housing Department, LCC. 
William Howell, Peter Carter et Colin St John Wilson. Cette réalisation était partie prenante de l'intense débat politico-culturel qui avait lieu alors au département du logement du LCC dans les années 1950: une rupture idéologique s'était créée entre ceux qui étaient favorables à l'esthétique du béton brut de l'Unité et ceux qui préféraient les surfaces en brique du New Empiricism (souvent appelé aussi le «People's Detailing »), présent dans les prototypes de logement conçus par Michael Powell, Cleeve Barr, Rosemary Stjerstedt et Oliver Cox. Ces derniers constituaient l'aile gauche, d'influence suédoise, du département du logement. Leurs préférences sociales et esthétiques allaient vers l'ethos pittoresque de la cité-jardin des New Towns de la première période ; autrement dit, leur modus operandi prolongeait la modernité populaire conçue dans l'esprit de conciliation du Festival of Britain (1951), qui revenait au valeurs sociales-démocrates de l'État-providence suédois, dans la mesure où elles avaient été implicitement reprises dans l'architecture de l'Exposition de Stockholm de 1930. Ce clivage entre une droite radicale et quasi-populiste et une gauche populiste sera par exemple mis en scène dans l'Alton West Estate de Roehampton où, aux petites unités disposées en chevrons réguliers (telles qu'elles avaient été projetées par l'équipe d'Howell), répondaient autant de tours issues directement des types de logements élevés inaugurés en Suède dans la ville nouvelle de Vällingby, et réalisés d'après les projets de Sven Markelius entre 1945 et 1954. Un schisme esthétique similaire s'était déjà produit pour le Royal Festival Hall, où la rigueur spatiale du plan libre corbuséen de Peter Moro (très apprécié par
Bentham Road

Housing Scheme, 1955.

Architectes Alan

Colquhoun, William

Howell, Peter Carter,

Colin St John Wilson.

(c) London County Council.

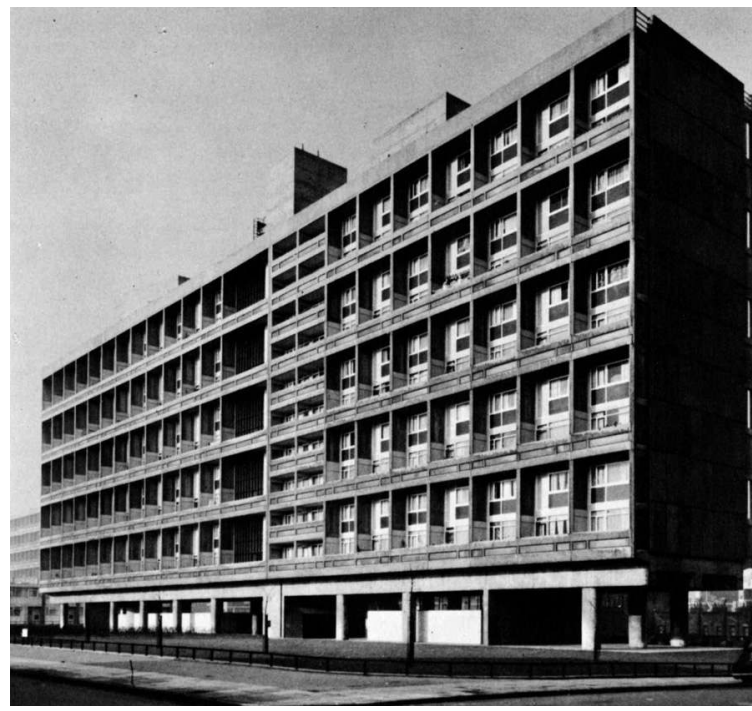


James Maude Richards dans son compte rendu de l'édifice en 1951) avait été rendue plus accessible et rassurante pour le grand public, grâce au mobilier en bois, aux ornements en cuivre, aux chaises en contre-plaqué courbé, aux tapis et tentures d'un style décoratif abstrait et coloré.

\section{Nouveau Brutalisme et vernaculaire " révisionniste " de la faible hauteur et de la haute densité}

La réinterprétation par Le Corbusier de l'architecture vernaculaire blanche, typique de la Méditerranée, dans son projet « Roq et Rob », avec sa voûte en berceau - un logement de haute densité projeté pour le Cap Martin en 1949 - a coïncidé avec la réévaluation par Thomas Sharp du village britannique traditionnel, dans son Anatomy of a Village de 1946, ayant influencé jusqu'à un certain point les propositions de logements villageois exposées par James Stirling et Alison et Peter Smithson au CIAM d'Aixen-Provence en 1953. Trois essais de Stirling du milieu des années 1950 témoignent du degré d'influence qu'exerça sur son travail la réhabilitation par Le Corbusier du mégaron méditerranéen à façade étroite, en tant que modèle valable pour le logement moderne. Ces essais, «From Garches to Jaoul » et « Le Corbusier and the Crisis of Rationalism », publiés dans The Architectural Review ${ }^{13}$ puis "Regionalism and Modern Architecture ", publié dans Architect's Year Book 7 en 1957, ont été l'expression du malaise de Stirling face à l'abandon par Le Corbusier de l'esthétique rationnelle, blanche et typique de l'ère de la machine, au profit d'une architecture vernaculaire modernisée, frisant le pittoresque. Et même si
Stirling avait adopté une approche comparable dans ses logements pour classes moyennes de Ham Common (Richmond, comté de Surrey, 1955) ou encore dans les logements populaires de Preston (Lancashire, 1957), tous deux construits en appareillage de brique, il redoutait sans cesse la faillite des constructeurs britanniques face aux prouesses techniques de l'industrie du bâtiment aux États-Unis: exemple manifeste de ces prouesses, la Lever House à New York - avec son ossature en acier inoxydable et ses murs-rideaux -, achevé la même année que I'Unité. Au regard du penchant de Le Corbusier pour la technique du béton brut, Stirling appréciait à contrecœur sa maîtrise de l'architecture néo-vernaculaire telle qu'elle apparaissait dans les voûtes de la Maison Jaoul, construite par des ouvriers algériens. Stirling note ainsi : « Les fenêtres ne sont pas là pour regarder au travers mais pour être regardées. L'œil, qui trouve un intérêt dans n'importe quelle surface, ne cherche pas, comme à Garches, à se consoler de la sévère finition à la texture indéterminée en examinant les contours et la forme du plan. "Cependant, au vu de sa propre pratique dans la première moitié des années 1950, Stirling était forcé de reconnaître, en dernière analyse, que l'adaptation ingénieuse par Le Corbusier de l'architecture vernaculaire à des fins modernes avait l'avantage d'engendrer un langage de formes plus accessible à la société en général. Cette technique et cette esthétique tranchaient sur le débat culturel qui se poursuivait au sein du département du logement du LCC et que Stirling avait résumé avec dédain par cette déclaration dont l'origine reste incertaine: "Il faut le reconnaître, William Morris était suédois!»
13. James Stirling, «From Garches to Jaoul: Le Corbusier as Domestic Architect in 1927 and 1953 ", The Architectural Review, $n^{\circ} 118$, septembre 1955, republié in Writings on architecture, recueil de textes édité par Robert Maxwell, Milan, Skira, 1998, pp. 29-40; "Ronchamp: Le Corbusier's chapel and the crisis of rationalism ", The Architectural Review, no 119, mars 1956, pp. 155-161, également republié in Writings [...],

pp. 41-50; " Regionalism and Modern Architecture », Architect's Year Book 7, Londres, 1957, également republié in Writings [...], pp. 173-178. 


\section{Theory and Design in the First Machine Age: Reyner Banham et Alan Colquhoun, 1960-1982}

Après avoir joué un rôle central au sein du département du logement du LCC, puis exercé dans un cabinet privé avec John Miller, Alan Colquhoun émergea comme critique d'envergure avec son compte rendu de l'ouvrage de Reyner Banham, Theory and Design in the First Machine Age, publié dans le British Journal of Aesthetics en janvier 1962. La réécriture radicale de I'histoire du Mouvement moderne par Banham, qui constituait initialement son doctorat à l'Institut Courtauld sous la direction de Pevsner, défiait l'exégèse néo-hégélienne qui avait jusque-là prédominé dans I'historiographie de I'architecture et de l'urbanisme modernes, notamment dans des ouvrages comme Pioneers of Modern Design: From William Morris to Walter Gropius ${ }^{14}$ de Pevsner, qui sortit en 1936, peu après le départ de celui-ci pour I'Angleterre en 1933, ou Space, Time and Architecture ${ }^{15}$ de Sigfried Giedion en 1941, ou encore Technics and Civilization $^{16}$ de Lewis Mumford en 1934, tous très influents sur la scène britannique d'après-guerre. Alors qu'il admettait la réévaluation anti-hégélienne de l'héritage idéologique du Mouvement moderne par Banham, Colquhoun souhaitait remettre en cause la polémique proto-futuriste et « technophile » de Banham. Comme il I'affirme vers la fin de sa critique de ce livre: "II est étrange qu'après avoir admis que certains édifices de la période sont des chefs-d'œuvre, M. Banham rejette les mystifications sans lesquelles ils n'existeraient pas. On peut se demander selon quels critères il juge d'un chefd'œuvre et comment il serait capable de démontrer qu'un édifice est simultanément un chef-d'œuvre et un échec. [...] Bien que M. Banham soit un historien trop scrupuleux pour s'engager dans certains mouvements ou en faveur de certaines personnalités, c'est clairement par les Futuristes et par Buckminster Fuller qu'il se sent le plus attiré. Il déplore l'absence, dans les plans de Le Corbusier, de toutes les qualités futuristes qu'il perçoit dans son organisation en différents éléments. Pourtant ces qualités "impures" existent chez les Futuristes euxmêmes et on ne peut guère nier que la gare d'Antonio Sant'Elia pour la "Città Nuova" révèle un parti pris parfaitement artistique. Faire ressortir l'académisme de Le Corbusier et le caractère dynamique d'un Futuriste, afin de montrer que le premier est arriéré et le second progressiste, nous semble être un procédé d'une validité historique douteuse ${ }^{17}$.»

Aussi affecté que Banham par l'empirisme britannique et aussi influencé que Colin Rowe par l'ouvrage antimarxiste de Karl Popper, The Open Society and its Enemies $^{18}$, Colquhoun aspirait à soumettre la culture architecturale moderne, théorique et pratique, à un examen plus rigoureux que ce qui avait prévalu jusque-là dans les pages de The Architectural Review, aux mains de polémistes du New Empiricism comme Lionel Brett et lan Nairn. Les écrits critiques de Colquhoun ont pu acquérir une portée particulièrement sensible et percutante avec son interprétation de la production de Le Corbusier, notamment à partir de son article du milieu des années 1960, "Formal and Functional Interactions: A Study of Two Late Buildings by Le Corbusier », publié dans Architectural Design en mai 1966. Le plus intéressant dans cette étude est la manière de traiter les deux projets
14. Nikolaus Pevsner, Pioneers of the Modern Movement: From William Morris to Walter Gropius, Londres, Faber et Faber, 1936.

15. Sigfried Giedion, Space, Time and Architecture, Harvard, Cambridge University Press, 1941 ; trad. fr. Espace, temps, architecture, [Bruxelles, Éditions de la Connaissance, 1968], Paris, Denoël, coll. « Médiations », 1978 (réimpr. 1990, 2004).
16. Lewis Mumford, Technics and Civilization, New York, Harcourt, 1934; trad. fr. Technique et Civilisation, Paris, Le Seuil, 1950.

17. Alan Colquhoun, «The Modern Movement in Architecture », British Journal of Aesthetics, vol. II, n 1, janvier 1962, pp. 59-65.
18. Karl Popper, The Open Society and its Enemies (vol. I: The Spell of Plato, vol. II: The High Tide of Prophecy: Hegel, Marx and the Aftermath), Londres, Routledge, 1945; trad. fr. La Société ouverte et ses ennemis (tome I: L'Ascendant de Platon, tome II: Hegel et Marx), Paris, Seuil, 1979. 
- I'ambassade de France à Brasilia et I'hôpital de Venise, datant tous deux de 1964 -, ce qui conduit Colquhoun à établir une distinction entre l'architecture comme objet de représentation publique et la construction comme processus d'accomodation matérielle, tout en reconnaissant que ces deux catégories culturelles ne sont jamais complètement séparées, même dans les cas où I'un ou l'autre aspect domine. Pour Colquhoun, alors que l'ambassade est composée de volumes et de surfaces simples, évoquant des "sensations constantes », à la base des tendances classiques de Le Corbusier, la forme de I'hôpital dérive quant à elle d'une opposition entre les deux dimensions susmentionnées. Celles-ci apparaissent comme une dialectique entre ses recherches de modèles de croissance - liées à son admiration de longue date pour les formes spontanées de l'architecture populaire et la nécessité de transformation directe d'un organisme dynamique en une forme aboutie. Toutefois, comme il l'affirme, « à y regarder de plus près, on s'apercevra qu'il y a polarisation de ces attitudes dans les deux projets et que chacun doit plus à son principe complémentaire qu'il $n^{\prime} y$ paraissait au premier abord ${ }^{19}$ ». En distinguant l'école de pensée structuraliste hollandaise, représentée par les travaux d'Aldo van Eyck et Herman Hertzberger, du modèle structurel apparemment semblable adopté par Le Corbusier dans son projet pour Venise, Colquhoun montre comment les répétitions isomorphes qui constituent l'orphelinat de Van Eyck à Amsterdam en 1960 sont sensiblement différentes du groupement structurel systémique adopté par Le Corbusier pour l'hôpital de Venise: " Ici, I'unité de base est en elle-même classée hiérarchiquement, avec des analogies biologiques plutôt que minérales; elle peut être modifiée localement sans que son principe en soit détruit. II se rapproche évidemment de projets matrice, tel celui de Candilis, Josic et Woods pour l'Université libre de Berlin. Le concept du plan de l'étage supérieur rappelle les medersas musulmanes d'Afrique du Nord, où des cellules de petites communautés d'étudiants sont regroupées autour de petites cours formant des systèmes satellites autour d'une cour centrale. Comme dans la medersa, l'ensemble domine les parties, et la nature répétitive du plan est contrôlée par une géométrie stricte ${ }^{20}$. "

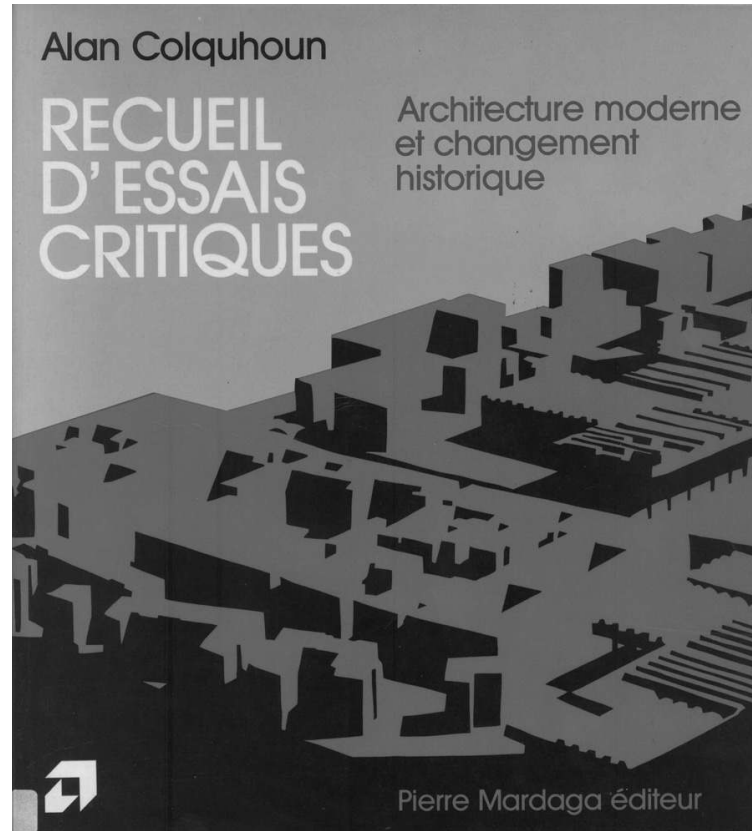

19. A. Colquhoun, «Formal and Functional Interactions: A Study of Two Late Buildings by Le Corbusier ", Architectural Design, vol. XXXVI, mai 1966, pp. 221-234; trad. fr. «Interactions formelles et fonctionnelles - étude de deux réalisations tardives de Le Corbusier "; A. Colquhoun, Architecture moderne et changement historique, Liège/Bruxelles, Pierre Mardaga, 1985, pp. 39-49. Citation p. 39.
20. A. Colquhoun, Recueil d'essais [...], op. cit. note 19, p. 46.

21. Ibid. p. 47.
Alan Colquhoun, Recueil d'essais critiques. Architecture moderne et changements historiques, Liège, Bruxelles, Pierre Mardaga éditeur, 1985. 
Colquhoun poursuit en notant que la forme de l'hôpital de Venise est soumise, comme la typologie des madrasas, à un contrôle harmonique et proportionnel des voûtes, et c'est ce qui, par-dessus tout, distingue l'approche de labyrinthe de Le Corbusier de la modularité répétitive du structuralisme hollandais. Même si l'hôpital de Venise était, comme le Centraal Beheer d'Hertzberger, un bâtiment sans façade au sens traditionnel du terme, il était, à la différence du Centraal Beheer, intégré au tissu interstitiel de la ville. Le projet était une véritable ville miniature, qui reflétait dans ses salles voûtées et ses couloirs intérieurs la morphologie médiévale rue/canal/campo de Cannareggio dans lequel il devait s'insérer. Comme le souligne Colquhoun, le projet de Le Corbusier pour l'hôpital de Venise possédait une complexité poétique presque toujours dans la plupart des édifices du XX $x^{e}$ siècle. À la fois habitat lacustre préhistorique, medersa, labyrinthe, phalanstère, hôpital et nécropole, le projet semble avoir été chargé, comme Colquhoun l'a observé prophétiquement, d'un ensemble d'interprétations « qui n'ont rien en commun avec une société dont les valeurs sont fondées sur les prétendues opinions de "I'homme moyen", aussi est-il possible qu'une telle machine à guérir ne soit pas comprise par le comité qui tient dans ses mains le destin du bâtiment ${ }^{21}$ ».

Cette observation aborde la question de la réception problématique de l'architecture moderne par la société, avec un franc-parler ironique peu habituel dans la critique contemporaine; elle témoigne de la subtilité de la critique de Colquhoun, capable de soutenir une position éthique sans tomber dans le moralisme. II affirme par exemple que « la critique occupe le no man's land entre l'enthousiasme et le doute, entre l'émotion poétique et I'analyse. Son but n'est, sauf dans des cas exceptionnels, ni de faire un panégyrique ni de condamner ${ }^{22}$. »Pourtant, comme il le précise encore, I'art n'est pas seulement un jeu gratuit; I'architecture doit plutôt être le résultat d'une réconciliation subtile et consciente mais pas moins disjonctive des différentes valeurs qui composent le monde contemporain.

Dans son essai publié en 1972, "Displacement of Concepts in Le Corbusier ${ }^{23}$, Colquhoun analyse la méthode de composition "déconstruction/reconstruction » adoptée par Le Corbusier et la façon d'utiliser cette méthode pour réconcilier certaines contradictions culturelles situées au cœur même du monde moderne, plus précisément la contradiction entre la typologie classique traditionnelle et la nécessité d'incorporer une instrumentalité empirique dans le corps de la forme moderne. Tel était le projet de synthèse original du purisme, qu'il soit appliqué à la peinture, à l'architecture ou au design industriel. Dans le même temps, il a essayé de répondre au postulat égalitaire de l'architecture moderne : comment combiner des éléments hétérogènes avec suffisamment d'habileté et de conviction pour gagner une large approbation sociale? Dans le cas de Le Corbusier, cela signifiait comment combiner l'autorité de l'ordonnance classique avec des références plus accessibles tirées de l'architecture populaire. Colquhoun montre que la stratégie d'inversion de Le Corbusier lui a permis de réinterpréter les paradigmes classiques de manière à les ouvrir vers l'assimilation d'éléments vernaculaires ou issus du monde de l'industrie. Cela a parfois conduit au déplacement des formes classiques, comme lorsque le péristyle

22. Ibid.

23. A. Colquhoun, «Displacement of Concepts in Le Corbusier ", Architectural Design, vol. XLIII, n 4, avril 1972, pp. 220 243. 
classique réapparaît dans l'architecture de Le Corbusier sous la forme du pilotis, les colonnes étant alors situées sous plutôt que devant l'édifice. L'intention était d'abord ici, le souligne Colquhoun, de bouleverser puis de faire une nouvelle proposition de la forme traditionnelle du " bottom, middle and top ". Cette hypothèse est confirmée par le fait que les « Cinq Points » d'une Architecture nouvelle de Le Corbusier intégraient originellement un sixième point, "l'élimination de la corniche ». Colquhoun fait valoir que - et il cite la villa Stein et le Pavillon Suisse comme exemples les plus frappants l'énergie plastique de la corniche allait se fondre dans la forme d'un parapet profond, dont la capacité première à consolider la composition était renforcée par l'emplacement d'une fenêtre centrale ou d'un oculus cyclopéen au sommet de la façade principale. De cette façon, le déplacement apparent du péristyle et de la corniche trouve un équivalent, à un niveau plus approfondi, dans la transformation du fenêtrage classique opérée par Le Corbusier. La fenêtre à la française, si appréciée par Auguste Perret, allait ainsi se trouver éclipsée par la fenêtre en longueur qui, avec son type industriel de fenêtre coulissante, bouleversait non seulement la forme de la traditionnelle porte-fenêtre, mais aussi son maniement. Cependant, comme le note Colquhoun avec raison, ce changement radical n'élimina pas complètement la fenêtre classique du répertoire initial du purisme, et des ouvertures étaient souvent utilisées également par Le Corbusier comme des accents dans la composition. II signale leur présence par exemple dans les couloirs d'accès du Pavillon Suisse où " l'introduction des fenêtres en longueur aurait été incompatible ${ }^{24} »$.

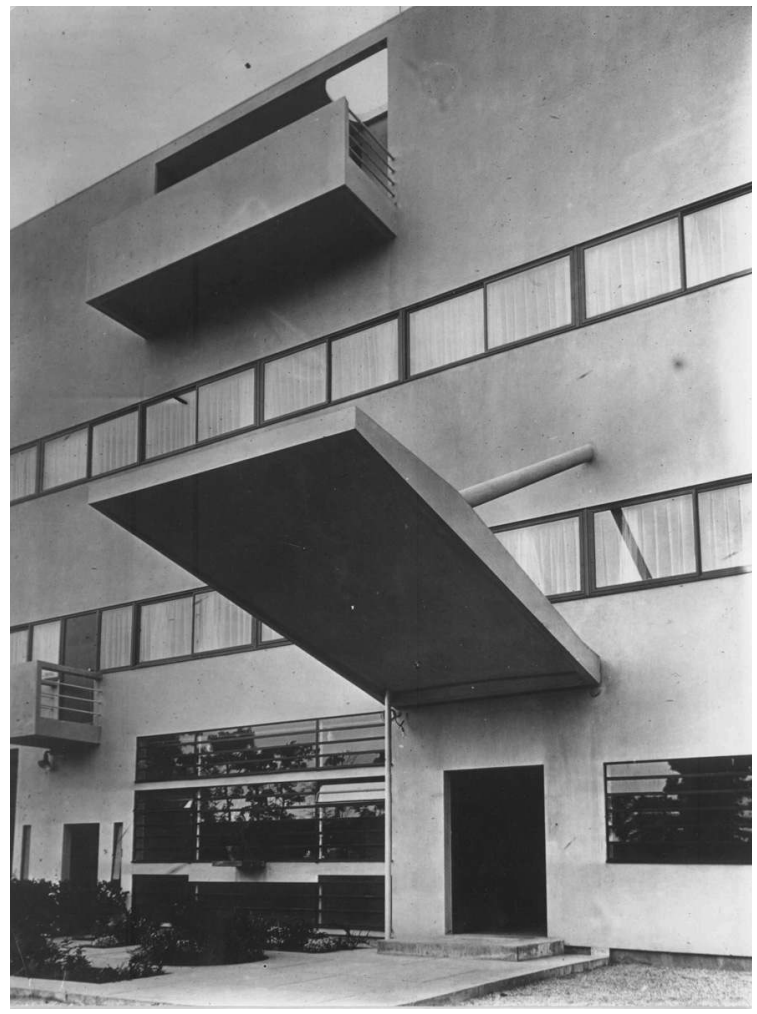

Villa Stein de Monzie, Garches. Le Corbusier (1926-1927). FLC L1(10)39. (C) FLC/ADAGP. 


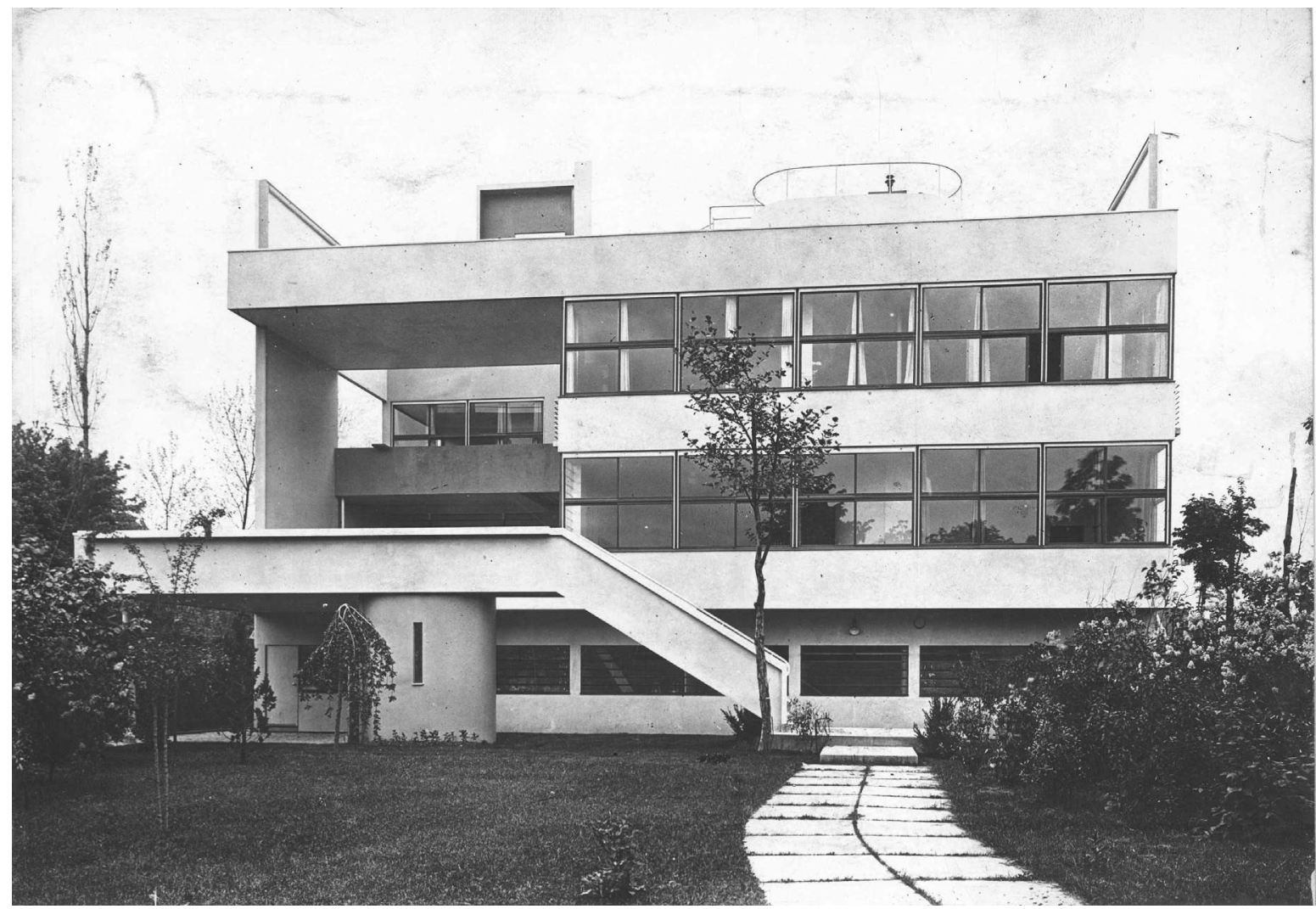

Villa Stein de Monzie,

Garches. Le Corbusier

(1926-1927). FLC L1(10)22.

(C) FLC/ADAGP. 
Après avoir observé comment Le Corbusier restreignait l'usage du plan libre au premier étage, traditionnellement hiérarchisé, Colquhoun montre comment les Cinq Points ne lui ont pas permis de combiner une enveloppe strictement classique avec le confort d'un intérieur vernaculaire. De cette façon, Le Corbusier répondait au défi d'Adolf Loos en réconciliant les valeurs rivales de la culture classique, des formes vernaculaires et de la technologie, à travers les subterfuges de la métaphore et du déplacement métonymique. Rétrospectivement, cette réconciliation doit sans doute être appréciée non seulement par rapport à l'époque de son ingénieuse création mais également par rapport aux confusions de la période présente. À propos du potentiel créatif de cette combinaison, Colquhoun a écrit: " La théorie architecturale a été dominée au cours de la dernière décennie par différentes formes de déterminisme et de populisme, dont aucune ne reconnaît que l'architecture constitue une entité culturelle en elle-même. Mais la matière première de l'architecture est, dans une large mesure, la culture architecturale à n'importe quel moment de son histoire. Tant que ces aspects de la création architecturale - aspects qui impliquent la transformation d'une culture existante - ne sont pas compris, nous n'allons pas réaliser une architecture capable de véhiculer des significations culturelles ${ }^{25}$. »

\section{Post-scriptum}

La réception critique largement autoréférentielle de Le Corbusier en Grande-Bretagne entre 1945 et 1972, par un petit cercle d'architectes, de critiques et d'historiens, a connu une sorte de conclusion post facto avec l'article d'Alan Colquhoun, "The Significance of Le Corbusier ».
Cet essai a été publié en 1984, soit dix-neuf ans après la mort du maître suisse, dans le premier volume des archives éditées par la Fondation Le Corbusier à Paris et par la Garland Publishing Company. Citons un extrait: « Le rôle idéologique et subversif que Le Corbusier a donné à I'architecture a lui-même été bouleversé par le développement "naturel" du capitalisme et sa récupération de l'avant-garde... Notre ambivalence par rapport à Le Corbusier reflète sa propre ambivalence envers le monde moderne et résulte des incertitudes de notre époque. D'une part, son concept de technocratie et sa vision de I'architecture comme un moyen de régénération morale et sociale semblent largement imparfaits. D'autre part, la subtilité plastique et le pouvoir métaphorique de ses édifices - leur originalité et la netteté de la touche - ne peuvent être niés. Pourtant sa grandeur incontestable en tant qu'architecte peut difficilement être dissociée de sa hauteur de vue et de la détermination impitoyable avec laquelle il l'a poursuivie. Si Le Corbusier fut induit en erreur à plusieurs titres, son illusion était celle du philosophearchitecte pour qui l'architecture, précisément à cause de l'idéal et du réel, est l'expression des vérités les plus profondes. II occupe l'un de ces rares moments de l'histoire où la vision de l'artiste et de l'homme passionné semble rejoindre le mythe collectif ${ }^{26}$."

Comme j'ai essayé de le montrer, à travers ce panorama qui se situe sur une courte période relativement localisée dans l'histoire de l'architecture moderne - à peine vingt-cinq années, et limitée essentiellement au débat autour d'une capitale -, la critique peut parfois prendre une forme féconde. Au sein de cette critique, il est alors assez facile de passer de la pratique de l'architecture à une

25. Ibid.

26. A. Colquhoun, «The Significance of Le Corbusier », in H. Allen Brooks, Le Corbusier, Princeton, Princeton University Press, 1987, pp. 17-26. 


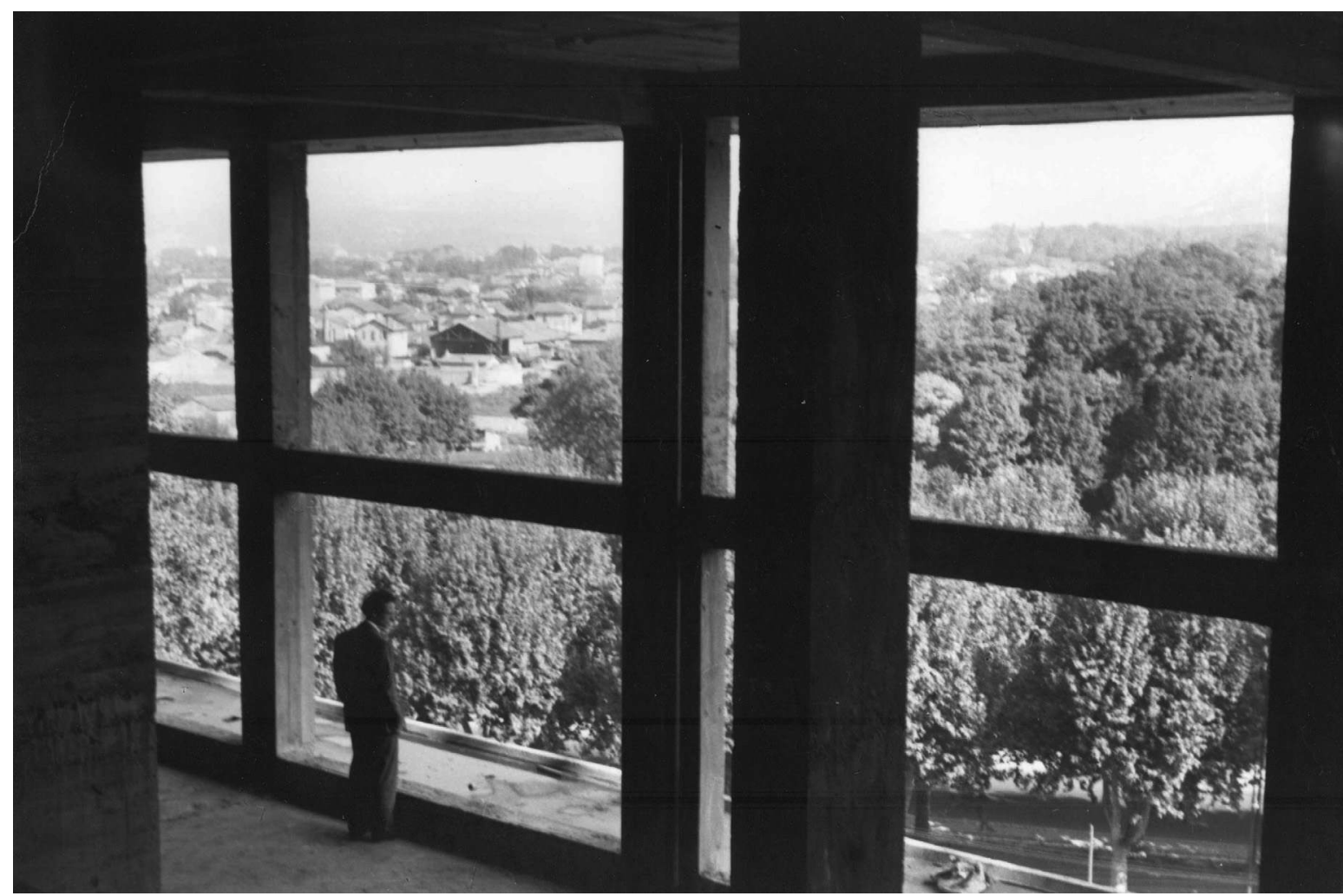

Unité d'habitation

de Marseille. Le Corbusier

(1945-1952). FLC L1(15)74.

(C) FLC/ADAGP. 
réflexion globale et simultanée sur la nature spécifique de cette pratique, permettant de favoriser des positions et des opinions approfondies et engagées ainsi qu'un potentiel pour toute une série d'interventions actives au niveau de la construction, des formes bâties. Dans un tel contexte, il est difficile de faire une distinction catégorique entre une critique polémique suscitée par des praticiens concurrents dans le feu de l'action, soutenus ou contestés par des critiques de service parfois hors du combat, et l'existence, en même temps, de théories censées faire preuve d'objectivité élaborées par des historiens/critiques et touchant à une pratique plus autonome de l'architecture en tant que telle. Il convient également de signaler la critique régulière de bâtiments déjà réalisés qui est faite dans les revues professionnelles, sans oublier une forme de critique plus générale et plus accessible que l'on trouve dans la presse dite populaire, deux types de critiques de plus en plus rares aujourd'hui, en une période plutôt dominée par le spectaculaire. II faut encore reconnaître qu'il existe aussi une critique d'un genre encore plus rare, celle faite par des écrivains/praticiens parfaitement cultivés et judicieux, capables de passer sans problème et en toute autonomie d'une considération sur le milieu concerné à une analyse sur le bien-fondé ou non d'un concept ou d'une pratique particulière par rapport aux questions fondamentales qui se posent au moment de la réalisation d'un bâtiment. Enfin, il y a cette idée qu'un projet architectural peut par lui-même proposer un paradigme critique qui touche aussi bien aux objectifs les plus larges de la pensée théorique et du débat politique qu'à un projet spécifique ou à une pratique hypothétique, susceptible d'être réalisés de façon concrète.
Terry Eagleton, dans le texte cité au début de cet essai, révèle que la critique conçue en un sens créatif et engagé ne peut exister qu'en lien avec la production culturelle et que lorsqu'une sphère publique aisément identifiable est présente à une échelle assez précise. Adrian Forty a écrit un article qui donne matière à réflexion, "Le Corbusier's British Reputation », à l'occasion du centenaire de ce dernier, célébré par l'école d'architecture de l'université de Cambridge - école britannique de premier plan dans les années 1960 - pour avoir été un architecte d'idées plus que le simple créateur d'un «style ». Ainsi que l'indique Forty: « Le Corbusier est important tout d'abord en tant que penseur spéculatif, avec une habilité hors du commun pour exprimer ses spéculations en termes de formes architecturales ${ }^{27}$. 》

C'est clairement ce qui a permis à son œuvre de stimuler un débat fécond en Grande-Bretagne pendant la période de l'État-providence de l'après-guerre qui, comme je l'ai suggéré, a pris fin avec la promulgation en 1972 d'un plan pour Milton Keynes, sans autre valeur que celle de l'économie de marché, très loin des urbanismes visionnaires projetés respectivement par Lubetkin et Le Corbusier. Notons que, malgré sa vague estime pour la poétique du Brutalisme, Banham verra toujours en Le Corbusier une figure totalement dépassée par la technologie. Ce jugement critique est confirmé, pour ainsi dire, par l'émergence triomphale de l'architecture high-tech sur la scène européenne, exposée au monde entier avec le concours international du Centre Georges Pompidou à Paris en 1972, année qui voit en effet la fin brutale de l'époque évoquée dans cet article.

TRADUCTION DE L'ANGLAIS PAR SOPHIE JACOTO
27. Adrian Forty, «Le Corbusier's British Reputation », in Tim Benton, Le Corbusier, Architect of the Century, catalogue de I'exposition à la Hayward Gallery, Londres, Arts Council of Great Britain, 1987, pp. 35-41. 